\title{
On Computation of the Bivariate Normal Distribution
}

\author{
By D. E. Amos*
}

Abstract. A quadrature and two series representations are given as limiting cases of a bivariate $t$-distribution. The quadrature is taken over the complementary error function and the series are sums of Bessel functions and incomplete beta functions, respectively. Comparisons with some known results are made in terms of accuracy and computer time.

The literature and tabulation of the bivariate normal distribution

$$
\begin{aligned}
N(h, k) & =\frac{1}{2 \pi\left(1-\rho^{2}\right)^{1 / 2}} \int_{-\infty}^{k} \int_{-\infty}^{h} \exp \left[-Q\left(x_{1}, x_{2}\right) / 2\right] d x_{1} d x_{2} \\
Q\left(x_{1}, x_{2}\right) & =\frac{x_{1}^{2}-2 \rho x_{1} x_{2}+x_{2}^{2}}{1-\rho^{2}}
\end{aligned}
$$

is extensive (see [7] and [11] for bibliographies). Quadratures developed by Sheppard [10] and Owen [9] stand out with respect to computational simplicity. The authors of [11] cite Sheppard's formula,

$$
\begin{aligned}
N(-h,-k) & =L(h, k) \\
& =\frac{1}{2 \pi} \int_{\cos ^{-1} \rho}^{\pi} \exp \left[-\left(h^{2}+k^{2}-2 h k \cos w\right) /\left(2 \sin ^{2} w\right)\right] d w, \\
& h>0, \quad k>0, \quad-1<\rho<1,
\end{aligned}
$$

tabulate $L(h, k)$ for $h \geqq 0, k \geqq 0$ and give function relations for various signs on $h$ and $k$. Owen's result is

$$
\begin{aligned}
N(h, k)= & \frac{1}{2}+\frac{1}{4}[\operatorname{erf}(h / \sqrt{ } 2)+\operatorname{erf}(k / \sqrt{ } 2)]-T\left(h, a_{h}\right)-T\left(k, a_{k}\right) \\
& -\left\{\begin{array}{l}
0 \quad h k>0 \text { or } h k=0 \text { and } h+k \geqq 0 \\
1 / 2 \quad \text { otherwise }
\end{array}\right.
\end{aligned}
$$

where

$$
T(h, a)=\frac{1}{2 \pi} \int_{0}^{a} \frac{\exp \left[-h^{2}\left(1+x^{2}\right) / 2\right]}{1+x^{2}} d x, \quad 0 \leqq a \leqq 1
$$

and

$$
a_{h}=\frac{-\rho+k / h}{\left(1-\rho^{2}\right)^{1 / 2}} \quad a_{k}=\frac{-\rho+h / k}{\left(1-\rho^{2}\right)^{1 / 2}}
$$

The relations

Received May 31, 1968, revised December 19, 1968.

* This work was supported by the United States Atomic Energy Commission. 


$$
\begin{gathered}
T(h,-a)=-T(h, a), \quad T(-h, a)=T(h, a) \\
T^{\prime}(h, a)=\frac{1}{4}[1+\operatorname{erf}(h / \sqrt{ } 2)]+\frac{1}{4}[1+\operatorname{erf}(a h / \sqrt{ } 2)], \quad a>1 \\
-\frac{1}{4}[1+\operatorname{erf}(h / \sqrt{ } 2)][1+\operatorname{erf}(a h / \sqrt{ } 2)]-T(a h, 1 / a)
\end{gathered}
$$

reduce the quadrature to a subset of $[0,1]$. The terms in brackets divided by 2 are cumulative normal probability integrals with arguments $h, k$, or $a h$.

In the text to follow, we develop a quadrature in terms of the coerror function and present two series representations for (1). We start by noting a bivariate $t$

$$
P\left(t_{1}, t_{2}\right)=\frac{1}{2 \pi\left(1-\rho^{2}\right)^{1 / 2}} \int_{-\infty}^{t_{2}} \int_{-\infty}^{t_{1}} \frac{d x_{1} d x_{2}}{\left[1+Q\left(x_{1}, x_{2}\right) / k\right]^{1+k / 2}}
$$

and a quadrature [2] for this distribution,

$$
\begin{aligned}
P^{P}\left(t_{1}, t_{2}\right)= & \frac{1}{2 \pi(k+1)\left[1+Q\left(t_{1}, t_{2}\right) / k\right]^{k / 2}} \\
& \times \int_{\theta_{1}}^{\theta_{2}} F\left(1, \frac{k}{2} ; \frac{k+3}{2} ; 1-c^{2} \cos ^{2}(\theta-\phi)\right) d \theta \\
& -\frac{\Gamma((k+1) / 2)}{\sqrt{ } \pi \Gamma(k / 2)\left[1+Q\left(t_{1}, t_{2}\right) / k\right]^{k / 2}} \int_{\theta_{1}}^{\theta_{2}} \frac{\delta(\theta) c \cos (\theta-\phi)}{\left[1-c^{2} \cos ^{2}(\theta-\phi)\right]^{(k+1) / 2}} d \theta
\end{aligned}
$$

where

$$
\begin{aligned}
& \delta(\theta)=\left\{\begin{array}{l}
0, \cos (\theta-\phi) \geqq 0 \\
1, \cos (\theta-\phi)<0
\end{array}, \quad c^{2}=\frac{Q\left(t_{1}, t_{2}\right) / k}{1+Q\left(t_{1}, t_{2}\right) / k},\right. \\
& \begin{array}{lll}
\theta_{1,2}=\pi \mp \tan ^{-1}\left(\frac{1+\rho}{1-\rho}\right)^{1 / 2}, & \phi=\tan ^{-1} \frac{\gamma_{2}}{\gamma_{1}}, & \gamma_{1} \geqq 0 \\
\phi=\pi+\tan ^{-1} \underline{\gamma_{2}} \gamma_{1}, & \gamma_{1}<0
\end{array}
\end{aligned}
$$

and

$$
\gamma_{1}=\frac{t_{1}+t_{2}}{(2(1+\rho))^{1 / 2}}, \quad \gamma_{2}=\frac{t_{2}-t_{1}}{(2(1-\rho))^{1 / 2}}, \quad Q\left(t_{1}, t_{2}\right)=\gamma_{1}{ }^{2}+\gamma_{2}{ }^{2}
$$

with $-\pi / 2<\tan ^{-1} z \leqq \pi / 2$ and $\phi=0$ if both $\gamma_{1}$ and $\gamma_{2}$ are zero. As $k \rightarrow \infty$, the bivariate $t$-distribution (4) goes to the cumulative bivariate normal (1). As a result, (5) goes to a corresponding formula for (1). The main tool in taking the limit is the analytic continuation formula for the (Gauss) hypergeometric function,

$$
\begin{aligned}
F\left(1, \frac{k}{2} ; \frac{k+3}{2} ; 1-x\right)= & (k+1) F\left(1, \frac{k}{2} ; \frac{1}{2} ; x\right) \\
& -2 \sqrt{ } \pi \frac{\Gamma((k+3) / 2)}{\Gamma(k / 2)} \sqrt{ } x(1-x)^{-(k+1) / 2}, \quad 0 \leqq x<1
\end{aligned}
$$

and the relations

$$
\begin{aligned}
\Phi\left(1, \frac{1}{2}, x\right) & =-e^{x} \sqrt{ } x \gamma\left(-\frac{1}{2}, x\right) / 2 \\
\gamma\left(-\frac{1}{2}, x\right) & =-2 \gamma\left(\frac{1}{2}, x\right)-2 e^{-x} / \sqrt{ } x \\
\Gamma(z+\alpha) / \Gamma(z+\beta) & \sim z^{\alpha-\beta} \text { for } z \rightarrow \infty
\end{aligned}
$$


where $\Phi$ is a confluent hypergeometric function. The result is

$$
\begin{aligned}
N\left(t_{1}, t_{2}\right)= & \frac{\left(\theta_{2}-\theta_{1}\right) e^{-Q / 2}}{2 \pi}-\frac{1}{2} \sqrt{\frac{Q}{2 \pi}} e^{-Q / 2} \\
& \times \int_{\theta_{1}-\phi}^{\theta_{2}-\phi} \exp \left[(Q / 2) \cos ^{2} \omega\right]|\cos \omega| \operatorname{erfc}\left(\sqrt{\frac{Q}{2}}|\cos \omega|\right) d \omega \\
& -\sqrt{\frac{Q}{2 \pi}} \int_{\theta_{1}-\phi}^{\theta_{2}-\phi} \exp \left[-(Q / 2) \sin ^{2} \omega\right] \delta(\omega) \cos \omega d \omega
\end{aligned}
$$

where

$$
\operatorname{erfc}(x)=\frac{2}{\sqrt{ } \pi} \int_{x}^{\infty} e^{-t^{2}} d t=1-\operatorname{erf}(x) \text { and } \delta(\omega)=\left\{\begin{array}{l}
0, \cos \omega \geqq 0 \\
1, \cos \omega<0
\end{array} .\right.
$$

A change of variables, $t=(Q / 2)$ sin $\omega$ reduces the second integral in (6) (when it applies) to sums or differences of error functions. Some approximations (Eq. 7.1.26 of [1, p. 299], see also [3] and [8]) not only produce erf $(x)$ but also give $e^{x^{2}}$ erfc (x) as a polynomial. This fact makes (6) competitive with (2) in a quadrature routine.

When high precision is required for erf $(x)$ and $e^{x^{2}}$ erfc $(x)$, the Chebyshev expansions of [3] and [8] can be used for approximations up to 20 figures. The backward recursive techniques of $[6]$ in the form

$$
\operatorname{erf}(x)=\left\{\begin{array}{ll}
\gamma\left(1 / 2, x^{2}\right) \\
1-i^{0} \operatorname{erfc}(x)
\end{array} \quad \operatorname{erfc}(x)= \begin{cases}1-\gamma\left(1 / 2, x^{2}\right) & 0 \leqq x \leqq \sqrt{ } 2 \\
i^{0} \operatorname{erfc}(x) & \sqrt{ } 2<x<\infty\end{cases}\right.
$$

also produce significant digits where $i^{n} \operatorname{erfc}(x), n=-1,0,1, \cdots$ is the iterated coerror function and, contrary to usual notation, the gamma function is normalized so that $\gamma(1 / 2, \infty)=1$. The table below gives a comparison of computer times for these procedures on a table of erf $(x)$ for $x=0(.1) 5$ :

$$
\frac{\text { Method }}{\text { Time (ms) }} \quad \frac{[1]}{25} \quad \frac{[3]}{179,59} \quad \frac{[6]}{321} \text {. }
$$

The NBS (Hastings) approximation 7.1.26 of [1] cites an absolute error bound of $1.5 \times 10^{-7}$. The series of [3] were truncated for six digits, and a relative error requirement of $1 \times 10^{-6}$ was set for the method of [6] with starting indices incremented by 20 . The Chebyshev polynomials required in [3] were computed according to

$$
T_{n}(x)=\cos \left(n \cos ^{-1} x\right), \quad n \geqq 0 .
$$

and

$$
T_{0}(x)=1, \quad T_{1}(x)=x, \quad T_{n+1}(x)=2 . x T_{n}(x)-T_{n-1}(x), \quad n \geqq 1
$$

respectively. In backward recursion, a parameter study of starting indices as a function of $x$ (for a given relative error) can reduce the time of computation by at least a factor of two by eliminating the need for repetitive evaluation in an error check. The break at $x=\sqrt{ } 2$ combines the efficiency (convergence) of the algorithm in each domain with the retention of significant digits.

Two other limiting cases of [2] are worth noting. As $k \rightarrow \infty$, formulae (10) and (11) of [2] go to 


$$
N\left(t_{1}, t_{2}\right)=\frac{e^{-Q / 2}}{2 \sqrt{ } \pi} \sum_{n=0}^{\infty} \frac{(-1)^{n} \delta_{n} S_{n}\left(\theta_{1}, \theta_{2}\right)}{2^{n} \Gamma((n+1) / 2)}\left(\frac{Q}{2}\right)^{n / 2} \Phi\left(\frac{n}{2}+1 ; n+1 ; \frac{Q}{2}\right)
$$

and

$$
N\left(t_{1}, t_{2}\right)=\frac{e^{-Q / 2}}{2 \sqrt{ } \pi} \sum_{n=0}^{\infty} \frac{(-1)^{n}}{\Gamma((n+1) / 2)}\left(\frac{Q}{2}\right)^{n / 2} \int_{\theta_{1}}^{\theta_{2}} \cos ^{n}(\theta-\phi) d \theta,
$$

respectively, where

$$
\begin{array}{ll}
\delta_{n}=1, n=0, & S_{n}\left(\theta_{1}, \theta_{2}\right)=\theta_{2}-\theta_{1}, n=0 \\
\delta_{n}=\frac{2}{n}, n>0, & S_{n}\left(\theta_{1}, \theta_{2}\right)=2 \sin \frac{n\left(\theta_{2}-\theta_{1}\right)}{2} \cos \frac{n\left(\theta_{1}+\theta_{2}-2 \phi\right)}{2}, n>0 .
\end{array}
$$

The relationship

$$
\begin{aligned}
\Phi(a+1,2 a+1 ; x) & =2^{a+1 / 2} \Gamma\left(a+\frac{1}{2}\right) \frac{d}{d x}\left\{e^{x / 2}(x / 2)^{-a+1 / 2} I_{a-1 / 2}\left(\frac{x}{2}\right)\right\} \\
& =2^{a-1 / 2} \Gamma\left(a+\frac{1}{2}\right) e^{x / 2}(x / 2)^{-a+1 / 2}\left[I_{a+1 / 2}\left(\frac{x}{2}\right)+I_{a-1 / 2}\left(\frac{x}{2}\right)\right]
\end{aligned}
$$

makes evaluation of the $\Phi$ functions in (7) practical since the backward recursion method described in [6] generates a sequence of Bessel functions $\left\{I_{\alpha+n}(z)\right\}$ almost as easily as a single one. The integral in (8) is the sum or difference of incomplete beta functions and an algorithm is described in [6] for these functions also.

The table below gives a comparison of average time per evaluation for a CDC 3600 computer based on 1064 cases. These cases comprise 56 points in the first quadrant of the $t_{1}, t_{2}$ plane with $.5 \leqq t_{1}{ }^{2}+t_{2}{ }^{2} \leqq 4$ and $\rho=-.9(.1) .9$.

$\frac{\text { Formula }}{\text { Average Time per Evaluation (ms) }} \quad \frac{(2)}{18.7} \quad \frac{(3)}{9.6} \quad \frac{(6)}{15.7} \quad \frac{(7)}{110} \quad \frac{(8)}{142}$.

A Romberg integration routine was used for (2), (3), and (6) with erf $(x)$ and $e^{x^{2}}$ erfc $(x)$ computed from NBS equation 7.1.26. A correction term

$$
\left[\operatorname{erf}\left(t_{1} / \sqrt{ } 2\right)+\operatorname{erf}\left(t_{2} / \sqrt{ } 2\right)\right] / 2
$$

for (2) is necessary to get first quadrant values of $N\left(t_{1}, t_{2}\right)$ from $L\left(t_{1}, t_{2}\right)$. In order to avoid slow convergence associated with a discontinuous derivative, two quadratures were summed for the first integral of (6) when $\cos \omega$ changed sign in the interval of integration. An absolute error check of $1 \times 10^{-5}$ was used for all formulae, with the truncated series incremented by 20 terms and the integration step halved. This produced at least six decimal place agreement between all formulae with seven decimal place agreement most common. Although 60 terms of (7) and 140 terms of (8) were required in extreme cases, 40 terms of each produced agreement to 8 decimal places in the majority of cases with 9 place agreement quite common. Backward recursive techniques were used for $e^{-x} I_{n}(x)$ and $I_{x}(n+1 / 2,1 / 2)$ in $(7)$ and $(8)$ respectively. Forms of these methods are given in [4] and [5].

Acknowledgment. The author wishes to acknowledge the assistance of Mr. George Elkins and Mrs. Sharon Daniel in coding the numerical experiments for the CDC 3600 compuțer. 
Applied Mathematics I)ivision

Sandia Corporation

Sandia Base

Albuquerque, New Mexico

1. M. Abramowitz \& I. A. Stegun, (Editors), Handbook of Mathematical Functions with Formulas, Graphs, and Mathematical Tables, Nat. Bur. Standards Appl. Math. Series, 55, Superintendent of Documents, U. S. Government Printing Office, Washington, I. C., 1964; 3rd printing, with corrections, 1965. MR 29 \#4914; MR 31 \#1400.

2. D. E. Amos \& W. G. Bulgren, "On the computation of a bivariate $t$-distribution," Math. Comp., v. 23, 1969, pp. 319-333.

3. C. W. Clenshaw, Chebyshev Series for Mathematical Functions, National Physical Lab. Math. Tables, vol. 5, HMSO, London, 1962. MR 26 \#362.

4. W. Gautschi, "Algorithm 222-incomplete beta function ratios," Comm. Assoc. Comput. Mach., v. 7, 1964, pp. 143-144; "Certification of algorithm 222," Ibid., v. 244, 1964.

5. W. Gautschi, "Algorithm 236-Bessel functions of the first kind," Comm. Assoc. Comput. Mach., v. 7, 1964, pp. 479-480; “Certification of algorithm 236," Ibid., v. 8, 1965, pp. 105-106.

6. W. GAUTSCHI, "Computational aspects of three-term recurrence relations," SIAM Rev., v. 9, 1967, pp. 24-82.

7. S. S. GUPTA, "Bibliography on the multivariate normal integrals and related topics," Ann. Math. Statist., v. 34, 1963, pp. 829-838. MR 27 \#2049.

8. Y. L. LUKE \& J. W IMP, "Jacobi polynomial expansions of a generalized hypergeometric function over a semi-infinite ray," Math. Comp., v. 17, 1963, pp. 395-404. MR 28 \#255.

9. D. B. OwEN, "Tables for computing bivariate normal probabilities," Ann. Math. Slatist., v. 27,1956, pp. $1075-1090$.

10. W. F. Sheppard, "On the calculation of the double integral expressing normal correlation," Trans. Cambridge Philos. Soc., v. 19, 1900, pp. 23-69.

11. Tables of the Bivariate Normal Distribution Function and Related Funclions, Nat. Bur. Standards Appl. Math. Series, 50, Superintendent of Documents, U. S. Government Printing Office, 1959. 